1 Hacettepe Journal of Mathematics and Statistics

$\bigcap$ Volume 45 (4) (2016), $1083-1090$

\title{
Morita equivalence based on Morita context for arbitrary semigroups
}

\author{
Hongxing Liu*
}

\begin{abstract}
In this paper, we study the Morita context for arbitrary semigroups. We prove that, for two semigroups $S$ and $T$, if there exists a Morita context $(S, T, P, Q, \tau, \mu)$ (not necessary unital) such that the maps $\tau$ and $\mu$ are surjective, the categories $U S$-FAct and $U T$-FAct are equivalent. Using this result, we generalize Theorem 2 in [2] to arbitrary semigroups. Finally, we give a characterization of Morita context for semigroups.
\end{abstract}

Keywords: semigroup, $S$-act, Morita context, functor, category.

2000 AMS Classification: 20M50

Received : 25.07.2015 Accepted : 16.09.2015 Doi : 10.15672/HJMS.20164520080

\section{Introduction}

Morita theory characterizes equivalences between module categories over rings with 1. Kyuno [5] studied Morita theory for rings without 1. Knauer [4] and Banschewski [1] independently generalized this theory to monoids. Banschewski [1] proved that for two semigroups $S$ and $T$, if the two categories $S$-Act and $T$-Act are equivalent, then $S$ is isomorphic to $T$. Talwar [8] extended Morita theory to semigroups with local units. He proved that for two semigroups with local units $S$ and $T$, the two categories $F S$-Act and $F T$-Act are equivalent $\Longleftrightarrow$ there is a unitary Morita context $(S, T, P, Q, \tau, \mu)$ such that the maps $\tau$ and $\mu$ are surjective, where $F S$-Act $=\{M \in S$-Act $\mid S M=M$ and $S \otimes$ $\left.\operatorname{Hom}_{S}(S, M) \cong M\right\}$. In [7], Talwar investigated strong Morita equivalence for factorisable semigroups. He got that if there is a unitary Morita context $(S, T, P, Q, \tau, \mu)$ such that the maps $\tau$ and $\mu$ are surjective, then $S$ and $T$ are strongly Morita equivalent. Chen and Shum [2] showed that, for factorisable semigroups $S$ and $T$, if there exists a unitary Morita context $(S, T, P, Q, \tau, \mu)$ such that the maps $\tau$ and $\mu$ are surjective, then the categories $U S$-FAct and $U T$-FAct are equivalent.

In this paper, we mainly use the techniques of paper [5] to study the corresponding problems for arbitrary semigroups. The paper is constructed as follows: In Section 2, we recall some basic notions; In Section 3, we give the main results of the paper. We prove

${ }^{*}$ School of Mathematical Sciences, Shandong Normal University, 250014, Jinan, P. R. China, Email: 1hxshanda@163.com 
that, for two semigroups $S$ and $T$, if there exists a Morita context $(S, T, P, Q, \tau, \mu$ ) (not necessary unital) such that the maps $\tau$ and $\mu$ are surjective, the categories $U S$-FAct and $U T$-FAct are equivalent. Also, we extend Theorem 2 in [2] to arbitrary semigroups. In Section 4, we give a characterization of Morita contexts for semigroups.

\section{Preliminaries}

Let $S$ be a semigroup. A set $M$ is a left $S$-act if there is a function from $S \times M$ to $M$, denoted $(s, m) \rightarrow s m$, such that $(s t) m=s(t m)(\forall s, t \in S, m \in M)$. If $M$ is a left $S$-act, we write ${ }_{S} M$. A left $S$-act $M$ is said to be unitary if $M=S M$. Similarly, we can define right acts over semigroups.

Let $M$ and $N$ be two $S$-acts. A map $f: M \rightarrow N$ is an $S$-morphism if $f$ satisfies $f(s m)=s f(m),(\forall m \in M, s \in S) . \operatorname{Let}_{\operatorname{Hom}_{S}}(M, N)$ denote the set of all $S$-morphisms from ${ }_{S} M$ to ${ }_{S} N$. Denote by $\operatorname{End}_{S}(M)$ the set of all $S$-morphisms from $M$ to itself. Let $S$-Act denote the category of left acts over a semigroup $S$.

The unital left $S$-acts together with the $S$-morphisms form a full subcategory of $S$-Act, which we shall denote by $U S$-Act.

Let $S$ and $T$ be two semigroups. An $S$-T-biact is a set $M$ which is both left $S$-act and right $T$-act and $(s m) t=s(m t)$ for all $s \in S, t \in T$ and all $m \in M$. A biact is said to be unitary if it is left and right unitary. If $M$ and $N$ are $S$-T-biact, a map $f: M \rightarrow N$ is called biact morphism if $f$ satisfies $f(s m)=s f(m)$ and $f(m t)=f(m) t$ for all $m \in M, s \in S, t \in T$.

Let $S$ be a semigroup and $M \in S$-Act. An equivalence $R$ on $S$ is a congruence if for all $s, t, a \in S$,

$$
(s, t) \in R \Rightarrow(a s, a t) \in R,(s a, t a) \in R .
$$

An equivalence $\rho$ on ${ }_{S} M$ is a congruence if for all $s \in S, m, n \in M$,

$$
(m, n) \in \rho \Rightarrow(s m, s n) \in \rho .
$$

If $\rho$ is a congruence on $M$, then $M / \rho$ is also a left $S$-act. The act $M / \rho$ is called a quotient act. Let $\epsilon$ be the identity congruence on $M$.

Let $S$ be a semigroup and $M \in S$-Act. According to [2], we use the following notations.

$$
\begin{gathered}
\zeta_{M}=\{(x, y) \in M \times M \mid s x=s y, \forall s \in S\} ; \\
U S \text {-FAct }=\left\{M \in U S \text {-Act } \mid \zeta_{M}=\epsilon\right\} .
\end{gathered}
$$

Obviously, $\zeta_{M}$ is a congruence on $M$.

For a right $S$-act $A_{S}$ and a left $S$-act ${ }_{S} B$, the tensor product $A \otimes_{S} B$ exists. In fact, $A \otimes_{S} B=(A \times B) / \sigma$, where $\sigma$ is the equivalence on $A \times B$ generated by

$$
\mathcal{R}=\{((x s, y),(x, s y)) \mid a \in A, b \in B, s \in S\} .
$$

We denote the element $(x, y) \sigma$ of $A \otimes_{S} B$ by $x \otimes y$.

By Proposition 1.4.10 of [3], we have that $a \otimes b=c \otimes d \Longleftrightarrow(a, b)=(c, d)$ or there is a sequence

$$
(a, b)=\left(x_{1}, y_{1}\right) \rightarrow\left(x_{2}, y_{2}\right) \rightarrow \cdots \rightarrow\left(x_{n}, y_{n}\right)=(c, d)
$$

such that either $\left(\left(x_{i}, y_{i}\right),\left(x_{i+1}, y_{i+1}\right)\right) \in T$ or $\left(\left(x_{i+1}, y_{i+1}\right),\left(x_{i}, y_{i}\right)\right) \in T$, where $1 \leq i \leq$ $n-1$.

If $A$ is a right $S$-act and $B$ is an $S$-T-biact, then $A \otimes_{S} B$ is a right $T$-act with

$$
(a \otimes b) t=a \otimes b t ;
$$

similarly, if $A$ is a $T$-S-biact and $B$ is a left $S$-biact, then $A \otimes_{S} B$ is a left $T$-act with

$$
t(a \otimes b)=t a \otimes b
$$

(Proposition 3.1, [8]). 


\section{Morita equivalence for semigroups}

In this section, $S$ and $T$ are arbitrary semigroups. If there exists a Morita context $(S, T, P, Q, \tau, \mu)$, we shall prove that the two categories $F: U S$-FAct $\rightleftharpoons U T$-FAct $: G$ are equivalent. Furthermore, if $(S, T, P, Q, \tau, \mu)$ is unital, we get that $F \cong(Q \otimes-) / \zeta_{(Q \otimes-)}$ and $G \cong(P \otimes-) / \zeta_{(P \otimes-)}$. This generalizes Theorem 2 in [2].

3.1. Definition. [8] Let $S$ and $T$ be two semigroups. If there exist sets $P$ and $Q$, such that

1) $P$ is an $S$-T-biact, $Q$ is a $T$-S-biact;

2) there are biact morphisms $\tau: P \otimes_{T} Q \rightarrow S$ and $\mu: Q \otimes_{S} P \rightarrow T$ written correspondingly as

such that

$$
\tau(p \otimes q)=<p, q>, \quad \mu(q \otimes p)=[q, p]
$$

$$
<p_{1}, q>\cdot p_{2}=p_{1} \cdot\left[q, p_{2}\right], \quad\left[q_{1}, p\right] \cdot q_{2}=q_{1} \cdot<p, q_{2}>
$$

for each $p, p_{1}, p_{2} \in P, q, q_{1}, q_{2} \in Q$. Then $(S, T, P, Q, \tau, \mu)$ is called a Morita context.

By Proposition 3.1 in [8], we have $\tau(p \otimes q) s=\tau((p \otimes q) s)=\tau(p \otimes q s)$, where $p \in$ $P, q \in Q, s \in S$. We will use this fact in the proof of Lemma 3.2 and Lemma 3.4.

3.2. Lemma. Let $(S, T, P, Q, \tau, \mu)$ be a Morita context, where $\tau$ and $\mu$ are surjective. Then

1) For all $M \in U S$-FAct, set $U=Q \times M$. Then $\widetilde{(Q, M)}=(Q \times M) / \rho_{(Q \times M)} \in U T$-FAct, where $\rho_{Q \times M}=\left\{\left((q, m),\left(q^{\prime}, m^{\prime}\right)\right) \in U \times U \mid \tau(p \otimes q) m=\tau\left(p \otimes q^{\prime}\right) m^{\prime}, \forall p \in P\right\}$.

2) For all $N \in U T$-FAct, set $V=P \times N$. Then $\widetilde{(P, N)}=(P \times N) / \rho_{(P \times N)} \in U T$-FAct, where $\rho_{P \times N}=\left\{\left((p, n),\left(p^{\prime}, n^{\prime}\right)\right) \in V \times V \mid \mu(q \otimes p) n=\mu\left(q \otimes p^{\prime}\right) n^{\prime}, \forall q \in Q\right\}$.

Proof 1) i) Clearly, $\rho_{U}$ is an equivalence on $U$. Set $(\widetilde{Q, M})=U / \rho_{U}$. Denote by $\overline{(r, m)}$ the equivalence class $(r, m) \rho_{U}$. For $t \in T$, we can write $t=\mu(q \otimes p)$ since $\mu$ is surjective.

For all $\overline{(q, m)} \in(\widehat{Q, M}), \mu\left(q^{\prime} \otimes p^{\prime}\right) \in T$, define

$$
\mu\left(q^{\prime} \otimes p^{\prime}\right) \overline{(q, m)}=\overline{\left(q^{\prime}, \tau\left(p^{\prime} \otimes q\right) m\right)} .
$$

If $\overline{\left(q_{1}, m_{1}\right)}=\overline{\left(q_{2}, m_{2}\right)}$, for all $p \in P$, we have $<p, q_{1}>m_{1}=<p, q_{2}>m_{2}$. Hence, the definition is independent of the choice of equivalence class representative.

If $\mu\left(q_{1} \otimes p_{1}\right)=\mu\left(q_{2} \otimes p_{2}\right)$, for all $x \in P$, we have

$$
\begin{aligned}
<x, q_{1}><p_{1}, q>m= & <x, q_{1}<p_{1}, q>>m=<x,\left[q_{1}, p_{1}\right] q>m \\
= & <x,\left[q_{2}, p_{2}\right] q>m=<x, q_{2}><p_{2}, q>m .
\end{aligned}
$$

Hence,

$$
\overline{\left(q_{1},<p_{1}, q>m\right)}=\overline{\left(q_{2},<p_{2}, q>m\right)}
$$

Therefore, the definition is well-defined.

For all $\mu\left(q_{1} \otimes p_{1}\right), \mu\left(q_{2} \otimes p_{2}\right) \in T, \overline{(q, m)} \in(\widetilde{Q, M})$, we have

$\left(\mu\left(q_{1} \otimes p_{1}\right) \mu\left(q_{2} \otimes p_{2}\right)\right) \overline{(q, m)}=\mu\left(\left[q_{1}, p_{1}\right] q_{2} \otimes p_{2}\right) \overline{(q, m)}=\overline{\left(\left[q_{1}, p_{1}\right] q_{2}, \tau\left(p_{2} \otimes q\right) m\right)}$ and

$\mu\left(q_{1} \otimes p_{1}\right)\left(\mu\left(q_{2} \otimes p_{2}\right) \overline{(q, m)}\right)=\mu\left(q_{1} \otimes p_{1}\right) \overline{\left(q_{2}, \tau\left(p_{2} \otimes q\right) m\right)}=\overline{\left(q_{1}, \tau\left(p_{1} \otimes q_{2}\right) \tau\left(p_{2} \otimes q\right) m\right)}$.

Then $\left(\mu\left(q_{1} \otimes p_{1}\right) \mu\left(q_{2} \otimes p_{2}\right)\right) \overline{(q, m)}=\mu\left(q_{1} \otimes p_{1}\right)\left(\mu\left(q_{2} \otimes p_{2}\right) \overline{(q, m)}\right)$. This means that $\widetilde{(\widetilde{Q, M})}$ is a left T-Act.

ii) Suppose $\left(\overline{(q, m)}, \overline{\left(q^{\prime}, m^{\prime}\right)}\right) \in \zeta_{(\widetilde{Q, M})}$. For all $y \in Q, x \in P$, we have

$$
\mu(y \otimes x) \overline{(q, m)}=\mu(y \otimes x) \overline{\left(q^{\prime}, m^{\prime}\right)} .
$$


That is,

This implies that

$$
\overline{(y, \tau(x \otimes q) m)}=\overline{\left(y, \tau\left(x \otimes q^{\prime}\right) m^{\prime}\right)} .
$$

$$
\tau(p \otimes y) \tau(x \otimes q) m=\tau(p \otimes y) \tau\left(x \otimes q^{\prime}\right) m^{\prime},
$$

for all $p \in P$. Since $M \in U S$-FAct, we have

$$
\tau(x \otimes q) m=\tau\left(x \otimes q^{\prime}\right) m^{\prime} .
$$

For arbitrary of $x$, we get that $\overline{(q, m)}=\overline{\left(q^{\prime}, m^{\prime}\right)}$.

iii) For all $m \in M$, since $M=S M$ and $\tau$ is surjective, we have $m=\tau\left(p \otimes q^{\prime}\right) m^{\prime}$, where $m^{\prime} \in M$. For all $\overline{(q, m)} \in(\widetilde{Q, M})$, we have

$$
\overline{(q, m)}=\overline{\left(q, \tau\left(p \otimes q^{\prime}\right) m^{\prime}\right)}=\mu(q \otimes p) \overline{\left(q^{\prime}, m^{\prime}\right)} \in T(\widetilde{Q, M}) .
$$

Hence, we get $T(\widetilde{Q, M})=\widetilde{(\widetilde{Q, M})}$. Therefore, $(\widetilde{Q, M}) \in U T$-FAct.

2) For all $\overline{(p, n)} \in \widehat{(P, N)}, \tau\left(p^{\prime} \otimes q^{\prime}\right) \in S$, define

$$
\tau\left(p^{\prime} \otimes q^{\prime}\right) \overline{(p, n)}=\overline{\left(p^{\prime}, \mu\left(q^{\prime} \otimes p\right) n\right)} .
$$

Similarly, we can prove $\widetilde{(P, N)} \in U S$-FAct.

3.3. Theorem. Let $S$ and $T$ be two semigroups. If $(S, T, P, Q, \tau, \mu)$ is a Morita context with $\tau$ and $\mu$ surjective, then we have the category equivalence $F: U S$-FAct $\rightleftharpoons U T$-FAct : $G$, where $F=(Q \times-) / \rho_{(Q \times-)}$ and $G=(P \times-) / \rho_{(P \times-)}$.

Proof Let $f: M \longrightarrow N$ be an $S$-morphism, where $M, N \in U S$-FAct. Define $\tilde{f}$ : $(\widetilde{Q, M}) \longrightarrow \widetilde{(Q, N)}$ by

$$
\tilde{f}(\overline{(q, m)})=\overline{(q, f(m))}
$$

Suppose $\overline{(q, m)}=\overline{\left(q^{\prime}, m^{\prime}\right)}$. For all $p \in P$, we have $\tau(p \otimes q) m=\tau\left(p \otimes q^{\prime}\right) m^{\prime}$. This implies that $f(\tau(p \otimes q) m)=f\left(\tau\left(p \otimes q^{\prime}\right) m^{\prime}\right)$. Since $f$ is an $S$-morphism, it follows that $\tau(p \otimes q) f(m)=\tau\left(p \otimes q^{\prime}\right) f\left(m^{\prime}\right)$. Hence, $\overline{(q, f(m))}=\overline{\left(q^{\prime}, f\left(m^{\prime}\right)\right)}$. This proves that $\tilde{f}$ is well-defined.

It is easy to check that $\tilde{f}$ is a left $T$-morphism.

Let $f: U \longrightarrow V$ and $g: V \longrightarrow W$ be two $S$-morphisms, where $U, V, W \in U S$-FAct. Let $\tilde{f}: \widetilde{(Q, U)} \longrightarrow \widetilde{(Q, V)}$ and $\tilde{g}: \widetilde{(Q, V)} \longrightarrow(\widetilde{Q, W})$ be $T$-morphisms determined by $f$ and $g$ respectively. Then $\widetilde{g f}=\tilde{g} \tilde{f}$. In fact, since $g f: U \longrightarrow W$ is an $S$-morphism, we have a $T$-morphism $\widetilde{g f}: \widetilde{(Q, U)} \longrightarrow(\widetilde{Q, W})$. This implies that $\operatorname{dom}(\widetilde{g f})=\widetilde{(Q, U)}=\operatorname{dom}(\tilde{g} \tilde{f})$. For all $\overline{(q, u)} \in \widetilde{(Q, U)}$, we have

$$
\widetilde{g f}(\overline{(q, u)})=\overline{(q, g f(u))}=\tilde{g} \overline{(q, f(u))}=\tilde{g} \tilde{f(q, u)} .
$$

Define $F: U S$-FAct $\longrightarrow U T$-FAct by $F(M)=(Q \times M) / \rho_{(Q \times M)}=(\widetilde{Q, M})$ and $F(f)=\tilde{f}$, for all $M, N \in U S$-FAct, $f \in \operatorname{Hom}_{S}(M, N)$. Then $F$ is a functor.

Similarly, for $U, V \in U T$-FAct, if $f: U \rightarrow V$ is a $T$-morphism, we can define $S$ morphism $\bar{f}: \widetilde{(P, U)} \longrightarrow \widehat{(P, V)}$ by

$$
\bar{f}(\overline{(p, u)})=\overline{(p, f(u))} .
$$

Also, for $U, V, W \in U T$-FAct, if $f: U \longrightarrow V$ and $g: V \longrightarrow W$ be two $T$-morphisms, then $\overline{g f}=\bar{g} \bar{f}$.

We can define a functor $G: U T$-FAct $\longrightarrow U S$-FAct by $G(N)=(P \times N) / \rho_{(P \times N)}=$ $\widetilde{(P, N)}$ and $G(f)=\bar{f}$, for all $N \in U T$-FAct, $g \in \operatorname{Hom}_{T}(M, N)$. 
For $M \in U S$-FAct, we have

$$
G F(M)=G((\widetilde{Q, M}))=(P, \widetilde{\widetilde{(Q, M}})) .
$$

Define $\eta_{M}: M \longrightarrow(P, \widetilde{(\widetilde{Q, M})})$ by

$$
\tau(p \otimes q) m \mapsto \overline{(p, \overline{(q, m)})}
$$

For all $p, p^{\prime} \in P, q, q^{\prime} \in Q, m, m^{\prime} \in M$, we have

$$
\begin{aligned}
& \tau(p \otimes q) m=\tau\left(p^{\prime} \otimes q^{\prime}\right) m^{\prime} \\
\Leftrightarrow & \tau(x \otimes y) \tau(p \otimes q) m=\tau(x \otimes y) \tau\left(p^{\prime} \otimes q^{\prime}\right) m^{\prime}, \\
& \text { for all } x \in P, y \in Q(\text { since } M \in U S \text {-FAct }) \\
\Leftrightarrow & \overline{(y, \tau(p \otimes q) m)}=\overline{\left(y, \tau\left(p^{\prime} \otimes q^{\prime}\right) m^{\prime}\right)}, \text { for all } y \in Q \\
\Leftrightarrow & \frac{\mu(y \otimes p)(q, m)}{(p, \overline{(q, m)})}=\overline{\left(p^{\prime}, \overline{\left(q^{\prime}, m^{\prime}\right)}\right)} .
\end{aligned}
$$

This shows that $\eta_{M}$ is well-defined and injective. It is obvious that $\eta_{M}$ is surjective. For $m \in M$, write $m=\tau\left(p^{\prime} \otimes q^{\prime}\right) m^{\prime}$, where $p^{\prime} \in P, q^{\prime} \in Q, m^{\prime} \in M$. For all $p \in P, q \in Q$, we have

$$
\begin{aligned}
\eta_{M}\left(\tau(p \otimes q) \tau\left(p^{\prime} \otimes q^{\prime}\right) m^{\prime}\right) & \left.=\overline{\left(p, \overline{\left(q, \tau\left(p^{\prime} \otimes q^{\prime}\right) m^{\prime}\right)}\right)}=\overline{\left(p, \mu\left(q \otimes p^{\prime}\right) \overline{\left(q^{\prime}, m^{\prime}\right)}\right.}\right) \\
& =\tau(p \otimes q) \overline{\left(p^{\prime}, \overline{\left(q^{\prime}, m^{\prime}\right)}\right)}=\tau(p \otimes q) \eta_{M}\left(\tau\left(p^{\prime} \otimes q^{\prime}\right) m^{\prime}\right) .
\end{aligned}
$$

Hence, $\eta_{M}$ is an $S$-isomorphism.

Let $f: M \longrightarrow N$ be an $S$-morphism. For $m=\tau(p \otimes q) m^{\prime} \in M$, we have

$$
\begin{aligned}
& G F(f) \eta_{M}(m)=G F(f) \eta_{M}\left(\tau(p \otimes q) m^{\prime}\right)=G F(f)\left(\overline{\left(p, \overline{\left(q, m^{\prime}\right)}\right)}\right) \\
& =\overline{\left(p, F(f)\left(\overline{\left(q, m^{\prime}\right)}\right)\right)}=\overline{\left(p, \overline{\left(q, f\left(m^{\prime}\right)\right)}\right)}=\eta_{N} f(m) \text {. }
\end{aligned}
$$

Hence, we have the following commutative diagram

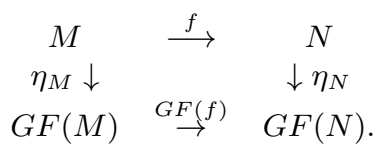

Therefore, $G F \cong 1_{U S-F A c t}$.

Similarly, we can prove that $F G \cong 1_{U T \text {-FAct }}$. This get the desired result.

3.4. Lemma. Let $(S, T, P, Q, \tau, \mu)$ be a Morita context and $M \in U S$-FAct. If $q_{1} \otimes m_{1}=$ $q_{2} \otimes m_{2} \in Q \otimes M$, we have $\overline{\left(q_{1}, m_{1}\right)}=\overline{\left(q_{2}, m_{2}\right)}$.

Proof 1) Suppose $\left(\left(q_{1}, m_{1}\right),\left(q_{2}, m_{2}\right)\right) \in T$. Without loss of generality, We suppose $q_{2}=q_{1} s, m_{1}=s m_{2}$, where $s \in S$. Then

$$
\tau\left(p \otimes q_{1}\right) m_{1}=\tau\left(p \otimes q_{1}\right) s m_{2}=\tau\left(p \otimes q_{1} s\right) m_{2},
$$

for all $p \in P$. Hence, we have $\overline{\left(q_{1}, m_{1}\right)}=\overline{\left(q_{1} s, m_{2}\right)}=\overline{\left(q_{2}, m_{2}\right)}$.

2) If $q_{1} \otimes m_{1}=q_{2} \otimes m_{2}$, By Proposition 1.4.10 of [3], we have that $\left(q_{1}, m_{1}\right)=\left(q_{2}, m_{2}\right)$ or for some positive integer $n>1$, there is a sequence

$$
\left(q_{1}, m_{1}\right)=\left(y_{1}, x_{1}\right) \rightarrow\left(y_{2}, x_{2}\right) \rightarrow \cdots \rightarrow\left(y_{n}, x_{n}\right)=\left(q_{2}, m_{2}\right)
$$

in which, for each $i$ in $\{1,2, \cdots, n-1\}$, either $\left(\left(y_{i}, x_{i}\right),\left(y_{i+1}, x_{i+1}\right)\right) \in \mathcal{R}$ or $\left(\left(y_{i+1}, x_{i+1}\right),\left(y_{i}, x_{i}\right)\right) \in \mathcal{R}$. By part 1$)$, we can easily get that $\overline{\left(q_{1}, m_{1}\right)}=\overline{\left(q_{2}, m_{2}\right)}$.

3.5. Definition. Let $S$ and $T$ be two semigroups. A Morita context $(S, T, P, Q, \tau, \mu)$ is called unital, if $P$ is a unital $S$-T-biact and $Q$ is a unital $T$-S-biact. 
3.6. Lemma. Let $(S, T, P, Q, \tau, \mu)$ be a unital Morita context and $M \in U S$-FAct. Then we have a $T$-isomorphism $(Q \times M) / \rho_{(Q \times M)} \cong(Q \otimes M) / \zeta_{(Q \otimes M)}$.

Proof Define a map $\varphi:(Q \times M) / \rho_{(Q \times M)} \rightarrow(Q \otimes M) / \zeta_{(Q \otimes M)}$ by $\varphi(\overline{(q, m)})=(q \otimes m) \zeta$, where $(q \otimes m) \zeta$ represent the congruence class $(q \otimes m) \zeta_{(Q \otimes M)}$.

Suppose $\overline{\left(q_{1}, m_{1}\right)}, \overline{\left(q_{2}, m_{2}\right)} \in \widehat{(\widehat{Q, M})}$. If $\overline{\left(q_{1}, m_{1}\right)}=\overline{\left(q_{2}, m_{2}\right)}$, we have $\tau\left(p \otimes q_{1}\right) m_{1}=$ $\tau\left(p \otimes q_{2}\right) m_{2}$, for all $p \in P$. Then

$\mu(y \otimes x)\left(q_{1} \otimes m_{1}\right)=\mu(y \otimes x) q_{1} \otimes m_{1}=y \otimes \tau\left(x \otimes q_{1}\right) m_{1}=y \otimes \tau\left(x \otimes q_{2}\right) m_{2}=\mu(y \otimes x)\left(q_{2} \otimes m_{2}\right)$,

for all $y \in Q, x \in P$. This implies that $\left(q_{1} \otimes m_{1}\right) \zeta=\left(q_{2} \otimes m_{2}\right) \zeta$. Therefore, $\varphi$ is welldefined. Obviously, $\varphi$ is surjective.

If $\left(q_{1} \otimes m_{1}\right) \zeta=\left(q_{2} \otimes m_{2}\right) \zeta$, for all $x \in P, y \in Q$, we have

$$
\mu(y \otimes x)\left(q_{1} \otimes m_{1}\right)=\mu(y \otimes x)\left(q_{2} \otimes m_{2}\right) .
$$

Since $y \otimes \tau\left(x \otimes q_{1}\right) m_{1}=y \tau\left(x \otimes q_{1}\right) \otimes m_{1}=\mu(y \otimes x)\left(q_{1} \otimes m_{1}\right)$, we get

$$
y \otimes \tau\left(x \otimes q_{1}\right) m_{1}=y \otimes \tau\left(x \otimes q_{2}\right) m_{2} .
$$

By Lemma 3.4, we have

$$
\overline{\left(y, \tau\left(x \otimes q_{1}\right) m_{1}\right)}=\overline{\left(y, \tau\left(x \otimes q_{2}\right) m_{2}\right)} .
$$

For all $p \in P$, we have

$\tau\left(\tau(p \otimes y) x \otimes q_{1}\right) m_{1}=\tau(p \otimes y) \tau\left(x \otimes q_{1}\right) m_{1}=\tau(p \otimes y) \tau\left(x \otimes q_{2}\right) m_{2}=\tau\left(\tau(p \otimes y) x \otimes q_{2}\right) m_{2}$.

Since $P$ is unitary and $\tau$ is surjective, we get

$$
\{\tau(p \otimes y) x \mid \text { for all } p, x \in P, q \in Q\}=S P=P .
$$

Then $\overline{\left(q_{1}, m_{1}\right)}=\overline{\left(q_{2}, m_{2}\right)}$. This proves that $\varphi$ is injective.

For all $\overline{(q, m)} \in(\widetilde{Q, M}), \mu(y \otimes x) \in T$, we have

$$
\begin{aligned}
\varphi(\mu(y \otimes x) \overline{(q, m)}) & =\varphi(\overline{(y, \tau(x \otimes q) m)})=(y \otimes \tau(x \otimes q) m) \zeta \\
& =(y \tau(x \otimes q) \otimes m) \zeta=(\mu(y \otimes x) q \otimes m) \zeta \\
& =\mu(y \otimes x)((q \otimes m) \zeta)=\mu(y \otimes x) \varphi(\overline{(q, m)}) .
\end{aligned}
$$

Hence, $\varphi$ is a $T$-isomorphism. That is, $(Q \times M) / \rho_{(Q \times M)} \cong(Q \otimes M) / \zeta_{(Q \otimes M)}$ as left $T$-act. $\square$

By Theorem 3.3 and Lemma 3.6, we have the following theorem which generalizes Theorem 2 in paper [2].

3.7. Theorem. Let $S$ and $T$ be two semigroups. If $(S, T, P, Q, \tau, \mu)$ be a unital Morita context with $\tau$ and $\mu$ are surjective, then we have the category equivalence $F: U S$-FAct $\rightleftharpoons$ $U T$-FAct : $G$, where $F=(Q \otimes-) / \zeta_{(Q \otimes-)}$ and $G=(P \otimes-) / \zeta_{(P \otimes-)}$.

\section{Characterization of Morita context}

In this section, we give an equivalent condition of Morita context in semigroup settings. Also, we give a characterization of Morita context for factorisable semigroups. Similar to Theorem 1 in [6], we have the following.

4.1. Theorem. Let $P$ and $Q$ be two sets. We have the following equivalent conditions.

1) There exist two semigroups $S$ and $T$ such that $(S, T, P, Q, \tau, \mu)$ is a Morita context.

2) There exist maps $\Gamma: P \times Q \times P \rightarrow P$ and $\Delta: Q \times P \times Q \rightarrow Q$ such that

I) $\Gamma\left(\Gamma\left(\left(p_{1}, q_{1}, p_{2}\right)\right), q_{2}, p_{3}\right)=\Gamma\left(\left(p_{1}, \Delta\left(\left(q_{1}, p_{2}, q_{2}\right)\right), p_{3}\right)\right)=\Gamma\left(p_{1}, q_{1}, \Gamma\left(\left(p_{2}, q_{2}, p_{3}\right)\right)\right)$;

II) $\Delta\left(\Delta\left(\left(q_{1}, p_{1}, q_{2}\right)\right), p_{2}, q_{3}\right)=\Delta\left(q_{1}, \Gamma\left(\left(p_{1}, q_{2}, p_{2}\right)\right), q_{3}\right)=\Delta\left(q_{1}, p_{1}, \Delta\left(\left(q_{2}, p_{2}, q_{3}\right)\right)\right)$. 
Proof 1) $\Rightarrow 2)$ : Suppose that $(S, T, P, Q, \tau, \mu)$ is a Morita context. Define $\Gamma: P \times$ $Q \times P \rightarrow P$ and $\Delta: Q \times P \times Q \rightarrow Q$ by putting $\Gamma\left(\left(p_{1}, q_{1}, p_{2}\right)\right)=\tau\left(p_{1} \otimes q_{1}\right) \cdot p_{2}$ and $\Delta\left(\left(q_{1}, p_{1}, q_{2}\right)\right)=\mu\left(q_{1} \otimes p_{1}\right) \cdot q_{2}$. We can easily check that $\Gamma$ and $\Delta$ satisfy the conditions I) and II).

$2) \Rightarrow 1):$ Define $H_{a}: P \rightarrow P$ by putting $H_{a}(p)=\Gamma((a, p))$ and define $K_{b}: Q \rightarrow Q$ by putting $K_{b}(q)=\Delta((b, q))$, where $a \in P \times Q$ and $b \in Q \times P$.

We write $\mathcal{X}=\left\{H_{a} \mid a \in P \times Q\right\}$ and $y=\left\{K_{b} \mid b \in Q \times P\right\}$. For all $H_{\left(p_{1}, q_{1}\right)}, H_{\left(p_{2}, q_{2}\right)} \in X$, for all $p \in P$, we have

$H_{\left(p_{1}, q_{1}\right)} H_{\left(p_{2}, q_{2}\right)}(p)=\Gamma\left(\left(p_{1}, q_{1}, \Gamma\left(p_{2}, q_{2}, p\right)\right)\right)=\Gamma\left(\left(\Gamma\left(p_{1}, q_{1}, p_{2}\right), q_{2}, p\right)\right)=H_{\left(\Gamma\left(p_{1}, q_{1}, p_{2}\right), q_{2}\right)}(p)$.

That is, $H_{\left(p_{1}, q_{1}\right)} H_{\left(p_{2}, q_{2}\right)}=H_{\left(\Gamma\left(\left(p_{1}, q_{1}, p_{2}\right)\right), q_{2}\right)} \in X$. Then we easily get that $X$ is a subsemigroup of $\operatorname{End}(P)$. Similarly, we have that $y$ is a subsemigroup of $\operatorname{End}(Q)$.

For all $p \in P, H_{a} \in X, K_{b} \in \mathcal{Y}$, define $H_{a} \cdot p=\Gamma((a, p))$ and $p \cdot K_{b}=\Gamma((p, b))$. Then $P$ is a $X$-y-biact. Similarly, for all $q \in Q$, we can define $K_{b} \cdot q=\Delta((b, q))$ and $q \cdot H_{a}=\Delta((q, a))$. This makes $Q$ to be a $y$-X-biact.

Now, we define $\alpha: P \otimes y Q \rightarrow X$ and $\beta: Q \otimes x P \rightarrow y$ by putting $\alpha(p \otimes q)=H_{(p, q)}$ and $\beta(q \otimes p)=K_{(q, p)}$, where $p \in P$ and $q \in Q$. It is easy to check that $\alpha$ and $\beta$ are both biact morphisms. Then

$$
\alpha\left(p_{1} \otimes q\right) \cdot p_{2}=H_{\left(p_{1}, q\right)} \cdot p_{2}=\Gamma\left(\left(p_{1}, q, p_{2}\right)\right)=p_{1} \cdot K_{\left(q, p_{2}\right)}=p_{1} \cdot \beta\left(q \otimes p_{2}\right) .
$$

Similarly, we have

$$
\beta\left(q_{1} \otimes p\right) q_{2}=q_{1} \alpha\left(p \otimes q_{2}\right) .
$$

Then $(X, y, P, Q, \alpha, \beta)$ is a Morita context.

4.2. Definition. [7] A semigroup $S$ is called factorisable if $S=S^{2}$.

4.3. Theorem. Let $P$ and $Q$ be two sets. We have the following equivalent conditions.

1) There exist two factorisable semigroups $S$ and $T$ such that $(S, T, P, Q, \tau, \mu)$ is a unital Morita context and $\tau$ and $\mu$ are surjective.

In this case, $(Q \otimes-) / \zeta_{(Q \otimes-)}: U S-$ Act $\rightleftharpoons U T-A c t:(P \otimes-) / \zeta_{(P \otimes-)}$ are equivalent functors.

2) There exist surjective maps $\Gamma: P \times Q \times P \rightarrow P$ and $\Delta: Q \times P \times Q \rightarrow Q$ satisfy the two conditions in part 2) of Theorem 4.1 and

III) For all $p, p^{\prime} \in P, q \in Q$, there exist $p_{1}, p_{2} \in P, q_{1}, q_{2} \in Q$ such that

$$
\Gamma\left(\left((p, q), p^{\prime}\right)\right)=\Gamma\left(\left(\Gamma\left(p_{1}, q_{1}, p_{2}\right), q_{2}, p^{\prime}\right)\right) .
$$

IV) For all $p \in P, q, q^{\prime} \in Q$, there exist $p_{1}, p_{2} \in P, q_{1}, q_{2} \in Q$ such that

$$
\Delta\left(\left((q, p), q^{\prime}\right)\right)=\Delta\left(\left(\Delta\left(q_{1}, p_{1}, q_{2}\right), p_{2}, q^{\prime}\right)\right) .
$$

Proof 1$) \Rightarrow 2)$ : Since $S$ is factorisable and $\tau$ is surjective, for all $p \in P, q \in Q$, there exist $p_{1}, p_{2} \in P, q_{1}, q_{2} \in Q$ such that $\tau(p \otimes q)=\tau\left(p_{1} \otimes q_{1}\right) \tau\left(p_{2} \otimes q_{2}\right)$. Hence,

$$
\begin{aligned}
\Gamma\left(\left((p, q), p^{\prime}\right)\right) & =\tau(p \otimes q) p^{\prime}=\tau\left(p_{1} \otimes q_{1}\right) \tau\left(p_{2} \otimes q_{2}\right) p^{\prime}=\tau\left(p_{1} \otimes q_{1}\right) \Gamma\left(\left(p_{2}, q_{2}, p^{\prime}\right)\right) \\
& =\Gamma\left(\left(p_{1}, q_{1}, \Gamma\left(p_{2}, q_{2}, p^{\prime}\right)\right)\right)=\Gamma\left(\left(\Gamma\left(\left(p_{1}, q_{1}, p_{2}\right)\right), q_{2}, p^{\prime}\right)\right) .
\end{aligned}
$$

Therefore, the condition III) holds. Similarly, we can get IV).

By Theorem 3.7 or Theorem 2 in [2], we have the category equivalence $(Q \otimes-) / \zeta_{(Q \otimes-)}$ : $U S$-Act $\rightleftharpoons U T$-Act $:(P \otimes-) / \zeta_{(P \otimes-)}$.

$2) \Rightarrow 1)$ : For all $H_{(p, q)} \in X, p^{\prime} \in P$, by the condition III), we have

$$
\Gamma\left(\left((p, q), p^{\prime}\right)\right)=\Gamma\left(\left(\Gamma\left(p_{1}, q_{1}, p_{2}\right), q_{2}, p^{\prime}\right)\right) .
$$


This implies that

$$
\begin{aligned}
H_{(p, q)}\left(p^{\prime}\right) & =\Gamma\left(\left((p, q), p^{\prime}\right)\right)=\Gamma\left(\left(\Gamma\left(p_{1}, q_{1}, p_{2}\right), q_{2}, p^{\prime}\right)\right) \\
& =\Gamma\left(\left(p_{1}, q_{1}, \Gamma\left(\left(p_{2}, q_{2}, p^{\prime}\right)\right)\right)\right)=H_{\left(p_{1}, q_{1}\right)} H_{\left(p_{2}, q_{2}\right)}\left(p^{\prime}\right) .
\end{aligned}
$$

That is, $H_{(p, q)}=H_{\left(p_{1}, q_{1}\right)} H_{\left(p_{2}, q_{2}\right)}$. This proves that $X$ is factorisable.

Similarly, we have that $y$ is a factorisable semigroup.

Since $\Gamma$ and $\Delta$ are surjective, we obviously have that $P$ and $Q$ are unital as biacts and $\alpha$ and $\beta$ are surjective. Hence, $(X, y, P, Q, \alpha, \beta)$ is a unital Morita context. $\square$

Acknowledgements The author is grateful to the referees for their valuable comments which improve the paper highly.

\section{References}

[1] Banaschewski, B. Functors into the categories of M-sets, Abh. Math. Sem. Univ. Hamburg 8, 49-64, 1972.

[2] Chen, Y. Q. and Shum, K. P. Morita equivalence for factorisable semigroups, Acta Mathematica Sinica (English Series), 17(3), 437-454, 2001.

3] Howie, J.M. Fundamentals of semigroup theory, Clarendon press, Oxford, 1995.

4] Knauer, U. Projectivity of acts and Morita equivalence of semigroups, Semigroup Forum 3, 359-370, 1972.

[5] Kyuno, S. Equivalence of module categories, Math. J. Okayama Univ, 28, 147-150, 1986.

[6] Paseka, J. Characterization of Morita equivalence pairs of quantales, Int. J. Theor. Phys., 44 (7),875-883, 2005.

[7] Talwar, S. Strong Morita equivalence and a generalisation of the Rees theorem, J. Algebra, 181, 371-394, 1996.

[8] Talwar, S. Morita equivalence for semigroups, J. Austral. Math. Soc. (Series A), 59, 81-111, 1995. 